\title{
Steel Catenary Riser Fatigue Life Prediction Using Linearized Hydrodynamic Models
}

\author{
C. Ebunuoha, C. V. Ossia* \\ Offshore Technology Institute, Faculty of Engineering, University of Port Harcourt, Port Harcourt, Nigeria \\ Email: *ossiacv@otiuniport.org
}

How to cite this paper: Ebunuoha, C. and Ossia, C.V. (2017) Steel Catenary Riser Fatigue Life Prediction Using Linearized Hydrodynamic Models. World Journal of Engineering and Technology, 5, 564-573. https://doi.org/10.4236/wjet.2017.54048

Received: August 9, 2017

Accepted: September 11, 2017

Published: September 14, 2017

Copyright $\odot 2017$ by authors and Scientific Research Publishing Inc. This work is licensed under the Creative Commons Attribution International License (CC BY 4.0).

http://creativecommons.org/licenses/by/4.0/ (c) (i) Open Access

\begin{abstract}
Steel catenary risers, (SCR) usually installed between seabed wellhead and floating platform are subjected to vortex shedding. These impose direct forces, hence cyclic stresses, and fatigue damage on the SCR. Riser failure has both economic and environmental consequences; hence the design life is usually greater than the field life, which is significantly reduced by vortex induced vibration (VIV). In this study, SCR and metOcean data from a field in Offshore Nigeria were substituted into linearized hydrodynamic models for simulations. The results showed that the hang off and touchdown regions were most susceptible to fatigue failure. Further analysis using Miner-Palm green models revealed that the fatigue life reduced from a design value of 20-years to 17.04-years, shortened by 2.96-years due to VIV. Furthermore, a maximum wave load of $5.154 \mathrm{kN}$ was observed. The wave loads results corroborated with those obtained from finite element Orca Flex software, yielding a correlation coefficient of 0.975 .
\end{abstract}

\section{Keywords}

Fatigue Life, Linearized Hydrodynamic Models, Steal Catenary Riser

\section{Introduction}

\subsection{Background}

The floating platform represents a new technology with promising future for the upstream oil and gas industries. Risers are used to interface the wellhead on the seabed to these floating surface production facilities. A steel catenary riser (SCR) is a rigid steel pipe of parabolic shape with considerable bending stiffness which is controlled mainly by weight, buoyancy and hydrodynamics forces due to currents and waves [1], [2]. They are typically 8 to 12 inches in diameter and oper- 
ate at a pressure of 2000 to 5000 Psi. It was first used at Gulf of Mexico (GoM) in 1994 and Gulf of Guinea (GoG) in 2004 [3]. It has greater bending stiffness, less flexibility and cost savings over flexible risers due to the low unit cost of steel pipe compared to flexible pipe.

It is therefore obvious that their failure is undesirable and associated with serious economic and environmental impacts. Curbing this requires the performance of detailed analyses of the riser using actual environmental load data. Environmental loads are the main source of dynamic excitation of offshore structures [4]. Due to the random nature of these loads, statistical properties consideration should be made when analyzing such structures [5]. Examples are loop currents in the GoM and highly directional environments in the GoG. Vessels motions and offsets have a major influence on riser analysis/modeling and should be considered. Environmental metOcean data used in SCR analysis are water depth, waves, currents, tide and surge variations and marine growth [6], [4]. It should be noted that the confidence with which metOcean data is derived varies considerably from one geographical location to another [7]. This has a large impact not only on the analysis of risers and mooring systems but also on the installation methods. This underscores the need for reliable site specific data [5]. It is recommended that currents specified for the riser analysis include an allowance for uncertainties in the data acquisition. Therefore, dynamic analysis is a handy solution that requires deep and comprehensive investigation of the associated systems [8], [9], [10], besides Couple analysis. Such dynamic analysis will give an excellent prediction of the riser behavior in unexpected weather conditions [4]. The phenomenon of vortex shedding of flexible cylindrical structures such as steel catenary risers (SCR) in water has received considerable attention. However, despite the robust experimental and theoretical data produced to trend performance, these studies are not exhaustive of all contributing factors. This study utilizes prevalent variable currents and wave data to predict in dynamic analyses to predict the total wave force (shear force), bending moment and fatigue life of a single deep water marine SCR in Offshore Nigeria.

\subsection{SCR Critical Fatigue Regions}

Vessel motions and hydrodynamic loading results in two critical fatigue regions of the SCR, namely:

1) At the vessel hang-off point (Tension Leg Deck) and

2) At the touch down zone (TDZ) (region of riser-soil interaction).

In the TDZ, cyclic motions of the SCR lead to complex interactions between the seabed and the riser. The severity of these interactions depends on the nature of the soil at the seabed for the geographical location involved. A SCR profile at the TDZ is shown in Figure 1.

According to Langer and Langer [13] the fatigue problem associated with the TDZ of a SCR may be more serious than is commonly understood, particularly for soft seafloor sediments typical of the GoM. The authors emphasized that for such soft sediments, a SCR will dig for itself a trench having a curved vertical 


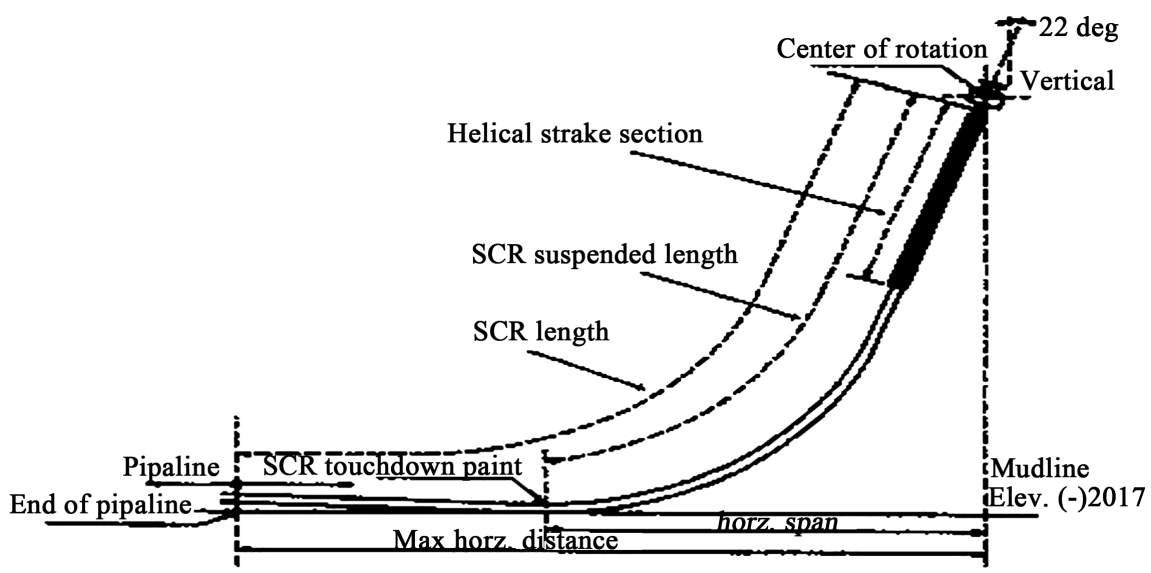

Figure 1. SCR profile at the touch down zone [11], [12].

profile that minimizes the shear forces in the SCR at the TDZ and consequently reduces the fatigue damage in the vicinity of the TDZ. The primary design criteria of SCRs are the usual internal and external pressure requirements and a requirement that the predicted minimum fatigue life of the riser must exceed the field life by a specified design factor. Fatigue damage is caused by cyclic bending of the riser, induced by the combination of platform motions and direct wave and current loadings on the riser [4] [14]. Fatigue analysis of an SCR involves counting the bending cycles that will be applied to the riser over the full range of the oceanographic loading conditions that will occur during its life time. The riser-soil interaction is complicated by the likelihood of extreme soil degradation in the TDZ during significant storms, with intervening periods where reconsolidation of the soil will occur.

\section{Materials and Methods}

\subsection{Procedure Work Flow}

The procedure adopted to estimate the wave forces on the modeled FPSO and SCR include:

* Physical modeling of FPSO and SCR;

* Select an appropriate wave theory (linearized waves, Morrisons equation or others, if necessary);

* Select the appropriate $C_{M}$ and $C_{D}$ based on Reynolds number and strouhal number, other factors;

* Perform bending moment analysis;

* Perform fatigue analyses;

Perform statistical analysis

\subsection{Physical Modeling}

Physical model is a larger or smaller physical copy of an object. It allows visualization for examining the model of information about the things the model represents. A physical OrcaFlex model of a single riser and floating production 
storage and offloading (FPSO) undergoing the Ochi-Hubble wave pattern typical to GoG is shown in Figure 2.

It is known that fluid motion past the SCR gives rise to Vortex shedding, which causes a lift force on the member at right angles to the fluid flow direction at a frequency corresponding to the eddy-shedding frequency. For steady flow past a cylinder (of diameter $D$ ) due to a current velocity $\left(U_{\max }\right)$ the main measure of the vortex shedding frequency $(f)$ of the forces is the strouhal number $(S)$ in Equation (1) which in turn depends on the Reynolds number $(R e)$ expressed as Equation (2).

$$
\begin{aligned}
S & =\frac{D f}{U_{\max }} \\
R e & =U_{\max } \frac{D}{\vartheta}
\end{aligned}
$$

where $v$-knematic viscosity of the fluid.

\subsection{Linearized Hydrodynamic Models}

By the concept of linear wave theory, the linearized hydrodynamic (Morrison) equation for calculating the total wave force $(\mathrm{F})$ is given by Equation (3).

$$
F=\frac{2 \pi \rho r H^{2} L}{T^{2}}\left[A_{1} C_{m} \sin \theta+A_{2} C_{d}|\cos \theta| \cos \theta\right]
$$

where $A_{1}$ and $A_{2}$-constants, $C_{d}$-drag coefficient, $C_{m}$-inertia coefficient, $L$-wavelength for intermediate water depth, $H$-significant wave height, $\rho$-water density, $r$-riser radius, $\theta$-theta in radians, $T$-period.

The total force $(F)$ in Equation (4) has two components, namely: 1) the drag component $\left(F_{d}\right)$ expressed as Equation (5) and 2) Inertia component $\left(F_{m}\right)$ expressed as Equation (6). The effect of marine growth was not considered in this study hence a drag coefficient value of $C_{d}=0.7$ and inertia coefficient value of $C_{m}=1.5$ was utilized in the models.

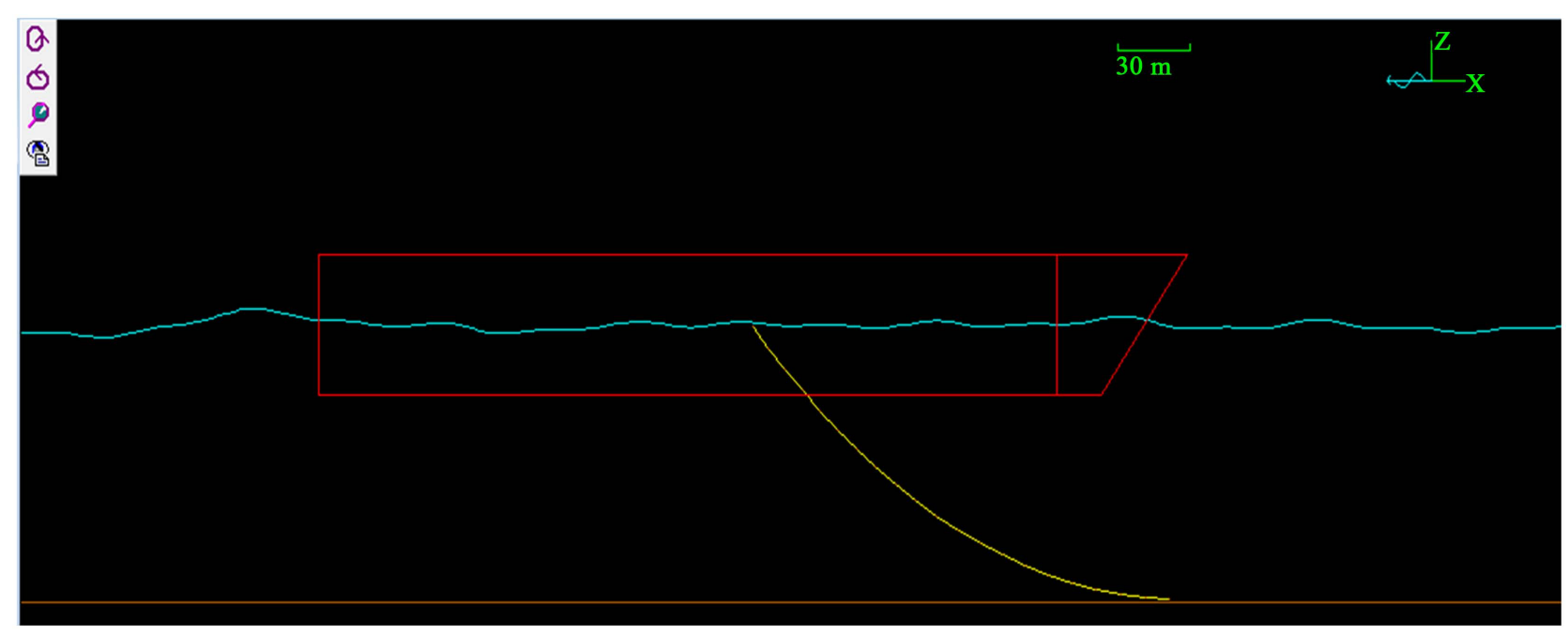

Figure 2. Physical OrcaFlex model of a riser and FPSO [4]. 


$$
\begin{gathered}
F=F_{d}+F_{m} \\
F_{d}=\frac{2 \pi \rho r H^{2} L}{T^{2}}\left[A_{2} C_{d}|\cos \theta| \cos \theta\right] \\
F_{m}=\frac{2 \pi \rho r H^{2} L}{T^{2}}\left[A_{1} C_{m} \sin \theta\right]
\end{gathered}
$$

The Morrison equation can also be expressed in terms of flow velocity $(U)$, flow acceleration $\left(\boldsymbol{U}^{\prime}\right)$, riser cross-sectional area $(A)$ and volume $(V)$ as in Equation (7).

$$
F=\rho C_{m} V U^{\prime}+\frac{1}{2} \rho C_{d} A U|U|
$$

where the drag force $F_{D}$ and inertia force $F_{m}$ are given by Equation (8) and Equation (9), respectively.

$$
\begin{gathered}
F_{d}=\frac{1}{2} \rho C_{d} A U|U| \\
F_{m}=\rho C_{m} V U^{\prime}
\end{gathered}
$$

The resulting overall bending moment $(M)$ of the structure is given by Equation (10).

$$
M=\frac{2 \pi \rho r H^{2} L^{2}}{T^{2}}\left[A_{3} C_{m} \sin \theta+A_{4} C_{d}|\cos \theta| \cos \theta\right]
$$

where $A_{3}$ and $A_{4}$-constants.

Similar to the total force, the resulting overall bending moment given in Equation (10) has two components, namely: 1) the drag component $\left(M_{d}\right)$ in Equation (11) and 2) the inertia component of moment $\left(M_{m}\right)$ in Equation (12).

$$
\begin{gathered}
M_{d}=\frac{2 \pi \rho r H^{2} L^{2}}{T^{2}} A_{4} C_{d}|\cos \theta| \cos \theta \\
M_{m}=\frac{2 \pi \rho r H^{2} L^{2}}{T^{2}}\left[A_{3} C_{m} \sin \theta\right]
\end{gathered}
$$

The wave equation constants $A_{1}$ and $A_{2}$ can be expressed as Equation (13) and Equation (14), respectively.

$$
\begin{gathered}
A_{1}=\frac{\pi r}{2 H} \\
A_{2}=\frac{1}{16 \sinh ^{2} k d}[2 k d+\sinh (2 k d)]
\end{gathered}
$$

where $k$-wave-number.

The overall bending moment equation constants $A_{3}$ and $A_{4}$ can be expressed by Equation (15) and Equation (16), respectively.

$$
\begin{gathered}
A_{3}=\frac{\pi r}{4 H \sinh (k d)}[1+(k d) \sinh (k d)-\cosh (k d)] \\
A_{4}=\frac{\pi r}{64 \sinh ^{2}(k d)}\left[2 k^{2} d^{2}+2(k d) \sinh (2 k d)+1-\cosh (2 k d)\right]
\end{gathered}
$$




\subsection{Fatigue Analysis}

The basic fatigue capacity ( $N$-number of cycles to failure) is expressed in terms of the shear stress $(S)$ as Equation (17).

$$
N=a S^{-m}
$$

where $a$ and $m$-empirical constants.

By taking the log of both sides, Equation (17) is reduced to Equation (18).

$$
\log N=\log a-m \log S
$$

The Miner-Palmgreen rule for the estimation of accumulated fatigue damage $\left(D_{f}\right)$ from number of stress cycles $(N)$ with variable range and stress due to shear force $\left(S_{i}\right)$ is given by Equation (19).

$$
D_{f}=\sum_{i} \frac{n\left(S_{i}\right)}{N\left(S_{i}\right)}
$$

where $n$-number of values.

According to Miner-Palmgreen rule, as used by Ebunuoha [4] the following accumulated fatigue damage criteria interpretation applies:

* $D_{f}>1$ implies that the damage is significant;

* $D_{f}<1$ implies that there is no damage;

* $D_{f}=1$ implies that there is imminent or impending damage.

\subsection{Statistical Analysis}

Bivariate data that measure two different variables are important and frequently encountered in statistical analysis. Correlation analysis provides techniques for analyzing the relation between two variables [15]. A less formal non-parametric approach to measure the extent of relationship between two such variables is the determination of Pearson's product moment of correlation coefficient $(r)$ [16] expressed as Equation (20).

$$
r=\frac{N \sum X Y-\left(\sum X\right)\left(\sum Y\right)}{\sqrt{\left[N \sum X^{2}-\left(\sum X\right)^{2}\right]\left[N \sum Y^{2}-\left(\sum Y\right)^{2}\right]}}
$$

where $X$-Representative of linear force values, $Y$-Representative of nonlinear force values, $N$-number of values.

\section{Results and Discussion}

\subsection{Inertia Force}

Figure 3 shows the variation of inertia force with water depth. The figure shows that the inertia force increases with increase in water depth. This is due to the fact that pressure increases with depth, following Pascal's principle of hydrostatic pressure.

\subsection{Drag Force}

The plot of drag force against water depth obtained is shown in Figure 4. The 


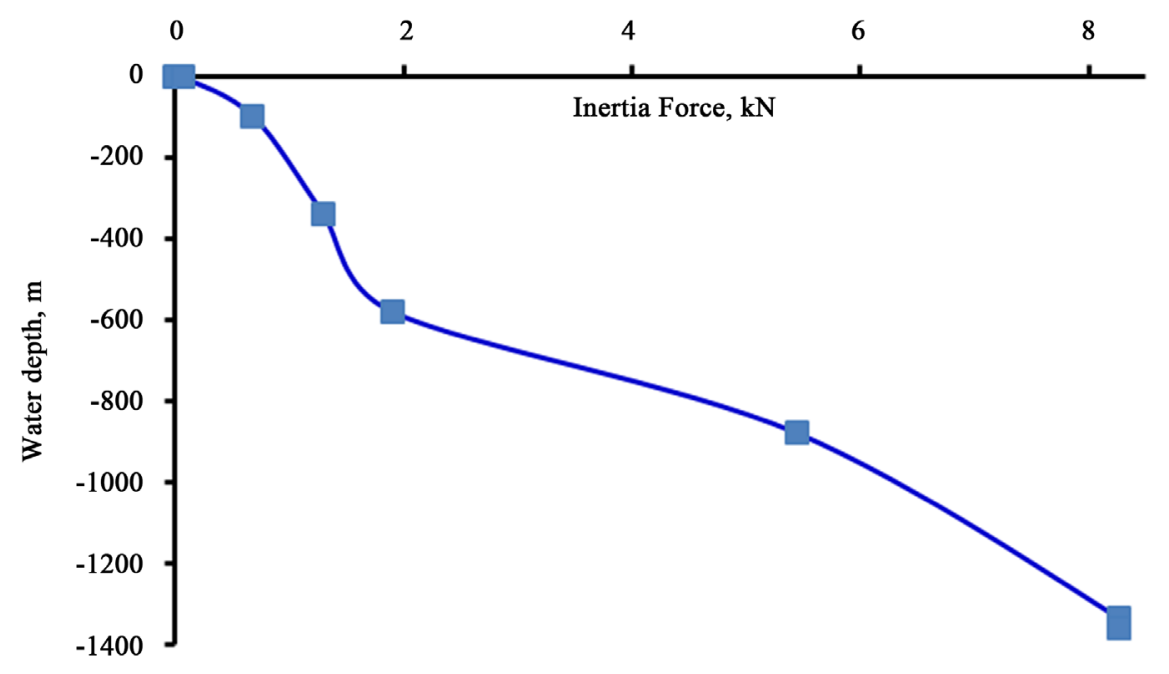

Figure 3. Inertia force variation with water depth.

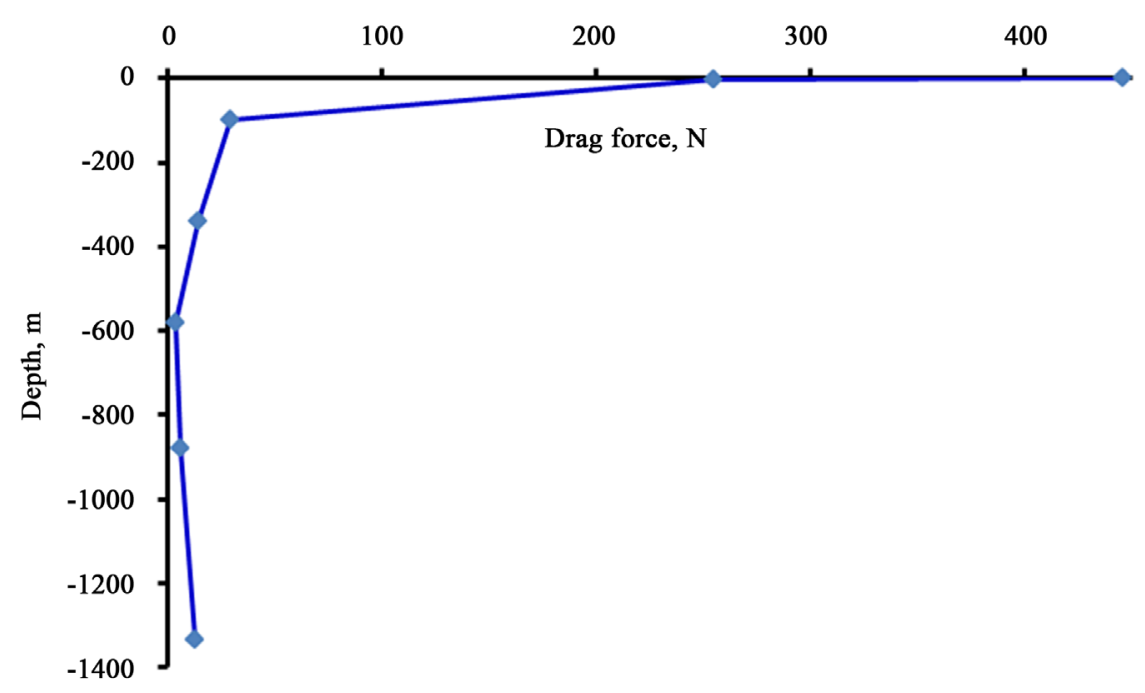

Figure 4. Drag force variation with water depth.

trend in the figure shows that the drag force decreases with increasing water depth. This is due to the current velocity which drastically reduces as water depth increases. At about $879 \mathrm{~m}$ it suddenly increased due to increased flow velocity in this depth. The continuous decrease is also associated with lower contribution of the wave drag force arising from the existence of waves at the air-water interface and near-surface region as the disturbance caused by this phenomenon decreases with water depth.

\subsection{Wave Force}

The trend of the total wave force with respect to water depth is shown in Figure 5. The trend shows that the total wave force increases with increasing water depth. It appeared to be higher at the hang off point $(6.446 \mathrm{kN})$ due to the additional tension there and highest towards the TDZ $(8.277 \mathrm{kN})$ due to the floating platform heave coupled with seabed interactions. 


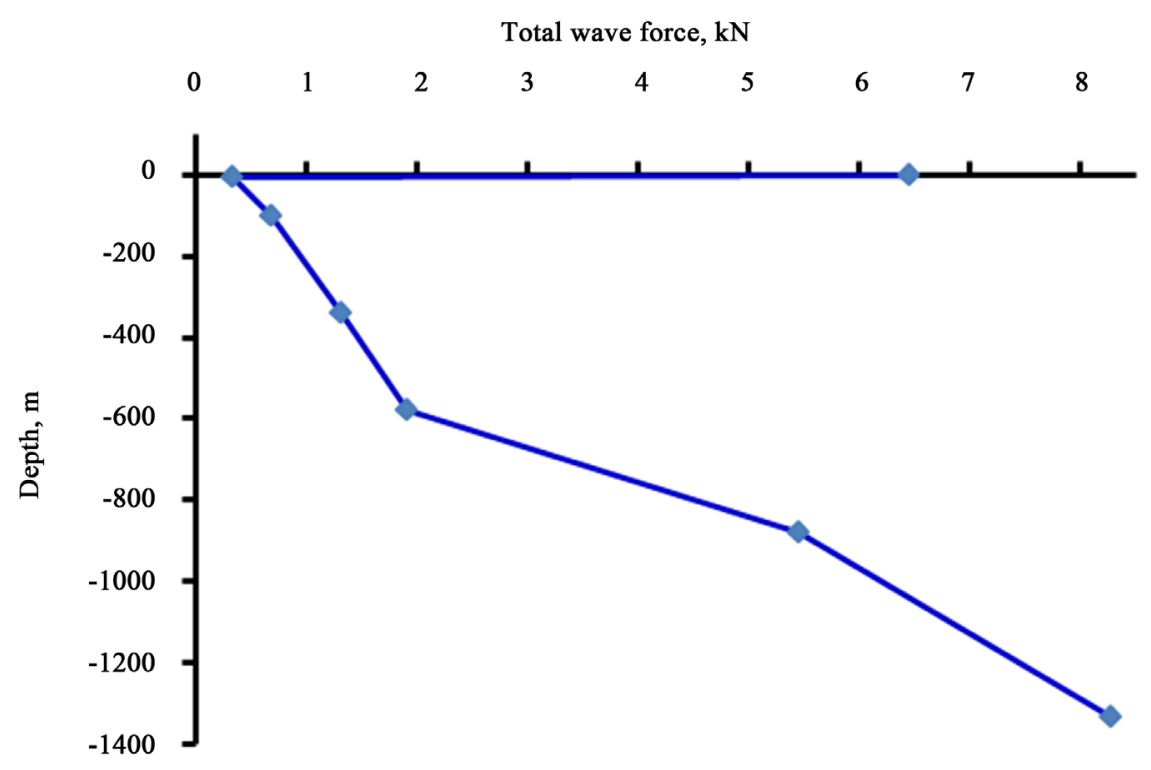

Figure 5. Total wave force variation with water depth.

\subsection{Bending Moment}

The plot of bending moment versus water depth is shown in Figure 6. The trend shows that the bending moment is highest at the TDZ (11.05 MNm). It is a clear indication that the touchdown zone is most vulnerable to failure due to soil-SCR structure interactions at this zone. It also showed that by tensioning the riser at the hang off point, the response of that SCR region to environmental loadings is reduced.

\subsection{Shear Force}

The combined plot of wave loads (shear force) against theta in radians is shown in Figure 7. It can be observed that there is maximum shear force (wave load) of $5.154 \mathrm{kN}$, occurring at about theta equal to 4.8 radians. Since the SCR fatigue design life is about 20 years, the remaining riser life is about 17.04 years. The results showed that VIV had shortened the SCR fatigue life by 2.96 years. There is $\pi$-radian phase lag between inertia component Fm and drag component Fd of the total force F.

\section{Conclusion}

The performance modeling of deep water steel catenary riser has been carried out. It was shown that the SCR structure operates in environments where it is adversely affected by varying conditions of currents and waves. These flow conditions subjects the riser to direct forces which results to cyclic stresses and with time culminates to fatigue damage. These led to the application of proven linearized hydrodynamic model to evaluate the deep water marine riser performance. MetOcean data were taken from a field in Offshore Nigeria. Simulations were carried out using OrcaFlex software. It revealed that hydrodynamic loadings results in two critical fatigue regions, namely: the vessel hang-off point and the 


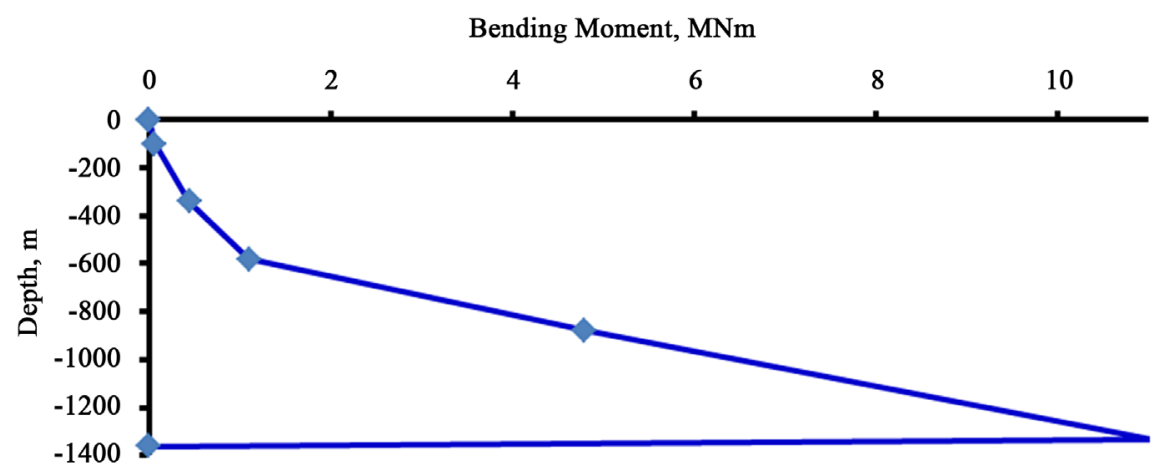

Figure 6. Bending moment variation with water depth.

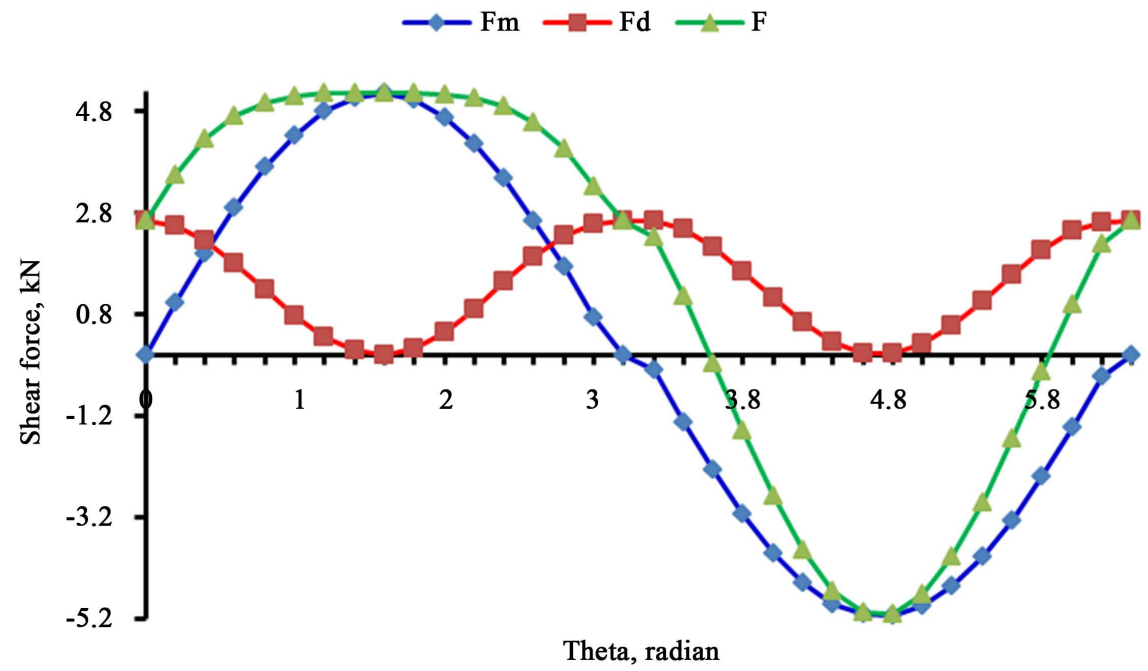

Figure 7. Shear force $(\mathrm{N})$ variation with Theta (radians) in one wave period.

TDZ-the area of soil-riser interaction. Also, a detailed fatigue analysis revealed that the remaining SCR life is 17.04-years, that is, 2.96-years less than the 20 -years design life. Furthermore, the statistical analysis carried out on total wave forces proved that both approach are highly correlated.

\section{References}

[1] Wajnikonis, C. and Robinson, R. (2000) Interactive Deepwater Riser Design, Analysis and Installation Methodology, IBP424200, Rio Oil and Gas Expo and Conference, 16-19 October 2000, Brazil, 4-8.

[2] Wajnikonis, C. and Leverette, S. (2009) Improvements in Dynamic Loading of Ultra Deep water Catenary Risers, OTC2009-20180, 4-7 May 2009, Houston, 1-7.

[3] Menglan, D., Jinghao, C. and Zhigang, L. (2011) Mechanics of Deepwater Steel Catenary Riser, Offshore Oil/Gas Research Center, China University of Petroleum, Beijing, 1-29.

www.intechopen.com/books/numerical-analysis-theory-and-application/mechanics -of-deepwater-steel-catenary-riser

[4] Ebunuoha, C. (2014) Performance Analysis of Deepwater Marine Riser. Unpublished M.Sc. Thesis Submitted to the Offshore Technology Institute, School of Graduate Studies, University of Port Harcourt, Nigeria, 1-100. 
[5] Michele, A.L.M., Andre, S.D. and Eduardo, S.S.S. (2009) Long-Term Fatigue and Extreme Design of Steel Risers. Proceedings of the ASME 2009, 28 th International Conference on Ocean, Offshore and Arctic Engineering, OMAE 2009-79272, 31 May-5 June 2009, Honolulu, 1-9.

[6] Chasparies, F., Modarres-Sadeghi, Y., Hover, F.S., Triantafyllou, M.S., Contantinides, Y. and Mukundan, H. (2009) VIV Hydrodynamics Data Extraction From Field Data. Proceedings of the ASME 2009, 28th International Conference on Ocean, Offshore and Arctic Engineering, OMAE 2009-79690, 31 May-5 June 2009, Honolulu, 1-6. https://doi.org/10.1115/OMAE2009-79690

[7] Faltinsen, O.M. (1990) Sea Loads on Ships and Offshore Structures. Cambridge University Press, 1-5.

[8] Brebbia, C.A. and Walker, S. (1979) Dynamic Analysis of Offshore Structures. Newness-Butterworths, London, 1-230.

[9] Low, Y.M. and Grime, A.J. (2011) Extreme Response Analysis of Floating Structures Using Coupled Frequency Domain Analysis. Journal of Offshore Mechanics and Arctic Engineering, 133, 200-209. https://doi.org/10.1115/1.4002734

[10] Low, Y.M. and Langley, R.S. (2008) Understanding the Dynamic Coupling Effects in Deepwater Floating Structures Using a Simplified Model. ASME Journal of Offshore Mechanics and Arctic Engineering, 130, 400-409.

[11] Chakrabarti, S.K. (2005) Hand Book of Offshore Engineering. Vol. 2, Elsevier Publications, 1-700.

[12] Shiri, H. (2014) Influence of Seabed Trench Formation on Fatigue Performance of Steel Catenary Risers in Touch down Zone. Elsevier Journal of Marine Structures, $36,1-20$.

[13] Langer, C.G. and Langer, C. (2003) Fatigue Life Improvement of Steel Catenary Risers due to Self-Trenching at the Touch down Point. OTC2003-15104, 5-8 May 2003, Houston, 1-11.

[14] Det Norske Veritas, DNV-RP-F 204 (2010) Riser Fatigue Rules and Standards. 1-34.

[15] Nwaogazie, I.L. (2011) Probability and Statistics for Science and Engineering Practice. De-Adroit Innovation, Enugu, 125-126.

[16] Mac'Odo, D.S. (1999) Statistics for Decision Making, Linnet Paul Publications, Port Harcourt. 190-191. 